\title{
Essential norm of generalized weighted composition operators from the Bloch space to the Zygmund space
}

\author{
Qinghua $\mathrm{Hu}^{1}$, Yafeng Shi ${ }^{2}$, Yecheng $\mathrm{Shi}^{3}$ and Xiangling Zhu ${ }^{4 *}$
}

*Correspondence: jyuzx|@163.com

${ }^{4}$ Department of Mathematics, Jiaying University, Meizhou,

Guangdong 515063, China Full list of author information is available at the end of the article

\begin{abstract}
In this paper, we give some estimates of the essential norm for generalized weighted composition operators from the Bloch space to the Zygmund space. Moreover, we give a new characterization for the boundedness and compactness of the operator.
\end{abstract}

MSC: $30 \mathrm{H} 30 ; 47 \mathrm{~B} 38$

Keywords: Bloch space; Zygmund space; essential norm; generalized weighted composition operator

\section{Introduction}

Let $X$ and $Y$ be Banach spaces. The essential norm of a bounded linear operator $T: X \rightarrow Y$ is its distance to the set of compact operators $K$ mapping $X$ into $Y$, that is,

$$
\|T\|_{e, X \rightarrow Y}=\inf \left\{\|T-K\|_{X \rightarrow Y}: K \text { is compact }\right\},
$$

where $\|\cdot\|_{X \rightarrow Y}$ is the operator norm.

Let $\mathbb{D}$ be the open unit disk in the complex plane $\mathbb{C}$ and $H(\mathbb{D})$ the space of analytic functions on $\mathbb{D}$. Let $\varphi$ be a nonconstant analytic self-map of $\mathbb{D}, u \in H(\mathbb{D})$, and $n$ be a nonnegative integer. The generalized weighted composition operator, denoted by $D_{\varphi, u}^{n}$, is defined on $H(\mathbb{D})$ by

$$
\left(D_{\varphi, u}^{n} f\right)(z)=u(z) f^{(n)}(\varphi(z)), \quad z \in \mathbb{D} .
$$

When $n=0$, the generalized weighted composition operator $D_{\varphi, u}^{n}$ is the weighted composition operator, denoted by $u C_{\varphi}$. In particular, when $n=0$ and $u=1$, we get the composition operator $C_{\varphi}$. If $n=1$ and $u(z)=\varphi^{\prime}(z)$, then $D_{\varphi, u}^{n}=D C_{\varphi}$, which was widely studied, for example, in [1-9]. If $u(z)=1$, then $D_{\varphi, u}^{n}=C_{\varphi} D^{n}$, which was studied, for example, in $[1,5,10,11]$. For the study of the generalized weighted composition operator on various function spaces see, for example, [12-21]. Recently there has been a huge interest in the study of various related product-type operators containing composition operators; see, e.g., $[22-30]$ and the references therein.

(c) $2016 \mathrm{Hu}$ et al. This article is distributed under the terms of the Creative Commons Attribution 4.0 International License (http://creativecommons.org/licenses/by/4.0/), which permits unrestricted use, distribution, and reproduction in any medium, provided you give appropriate credit to the original author(s) and the source, provide a link to the Creative Commons license, and indicate if changes were made. 
The Bloch space, denoted by $\mathcal{B}$, is defined to be the set of all $f \in H(\mathbb{D})$ such that

$$
\|f\|_{\mathcal{B}}=|f(0)|+\sup _{z \in \mathbb{D}}\left(1-|z|^{2}\right)\left|f^{\prime}(z)\right|<\infty
$$

$\mathcal{B}$ is a Banach space with the above norm. An $f \in \mathcal{B}$ is said to belong to the little Bloch space $\mathcal{B}_{0}$ if $\lim _{|z| \rightarrow 1}\left|f^{\prime}(z)\right|\left(1-|z|^{2}\right)=0$. See [31] for more information of Bloch spaces. Composition operators, as well as weighted composition operators mapping into Bloch-type spaces were studied a lot see, for example, [3, 6, 16, 32-45].

The Zygmund space, denoted by $\mathcal{Z}$, is the space consisting of all $f \in H(\mathbb{D})$ such that

$$
\|f\|_{\mathcal{Z}}=|f(0)|+\left|f^{\prime}(0)\right|+\sup _{z \in \mathbb{D}}\left(1-|z|^{2}\right)\left|f^{\prime \prime}(z)\right|<\infty
$$

It is easy to see that $\mathcal{Z}$ is a Banach space with the above norm $\|\cdot\|_{\mathcal{Z}}$. See $[4,7,12,15,16$, $22,36,46-50]$ for some results of the Zygmund space and related operators mapping into the Zygmund space or into some of its generalizations.

In 1995, Madigan and Matheson proved that $C_{\varphi}: \mathcal{B} \rightarrow \mathcal{B}$ is compact if and only if (see [38])

$$
\lim _{|\varphi(z)| \rightarrow 1} \frac{\left(1-|z|^{2}\right)}{\left(1-|\varphi(z)|^{2}\right)}\left|\varphi^{\prime}(z)\right|=0
$$

In 1999, Montes-Rodrieguez in [40] obtained the exact value for the essential norm of the operator $C_{\varphi}: \mathcal{B} \rightarrow \mathcal{B}$, i.e.,

$$
\left\|C_{\varphi}\right\|_{e, \mathcal{B} \rightarrow \mathcal{B}}=\lim _{s \rightarrow 1} \sup _{|\varphi(z)|>s} \frac{\left(1-|z|^{2}\right)\left|\varphi^{\prime}(z)\right|}{\left(1-|\varphi(z)|^{2}\right)} .
$$

Tjani in [43] proved that $C_{\varphi}: \mathcal{B} \rightarrow \mathcal{B}$ is compact if and only if $\lim _{|a| \rightarrow 1}\left\|C_{\varphi} \sigma_{a}\right\|_{\mathcal{B}}=0$, where $\sigma_{a}=\frac{a-z}{1-\bar{a} z}$. Wulan et al. in [44] showed that $C_{\varphi}: \mathcal{B} \rightarrow \mathcal{B}$ is compact if and only if $\lim _{j \rightarrow \infty}\left\|\varphi^{j}\right\|_{\mathcal{B}}=0$. Ohno et al. studied the boundedness and compactness of the operator $u C_{\varphi}$ on the Bloch space in [41]. The estimate for the essential norm of the operator $u C_{\varphi}$ on the Bloch space was given in [37]. Some new estimates for the essential norm of $u C_{\varphi}$ on the Bloch space were given in [33, 39]. In [21], Zhu has obtained some estimates for the essential norm of $D_{\varphi, u}^{n}$ on the Bloch space when $n$ is a positive integer.

Stević studied the boundedness and compactness of $D_{\varphi, u}^{n}: \mathcal{B} \rightarrow \mathcal{Z}$ in [16] (see also [50]). In [12], Li and Fu obtained a new characterization for the boundedness, as well as the compactness for $D_{\varphi, u}^{n}: \mathcal{B} \rightarrow \mathcal{Z}$ by using three families of functions. We combine the results in [12] and [16] as follows.

Theorem $\mathbf{A}$ Let $n$ be a positive integer, $u \in H(\mathbb{D})$, and $\varphi$ be an analytic self-map of $\mathbb{D}$. Suppose that $D_{\varphi, u}^{n}: \mathcal{B} \rightarrow \mathcal{Z}$ is bounded, then the following statements are equivalent:

(a) The operator $D_{\varphi, u}^{n}: \mathcal{B} \rightarrow \mathcal{Z}$ is compact.

(b)

$$
\lim _{|\varphi(w)| \rightarrow 1}\left\|D_{\varphi, u}^{n} f_{\varphi(w)}\right\|_{\mathcal{Z}}=\lim _{|\varphi(w)| \rightarrow 1}\left\|D_{\varphi, u}^{n} g_{\varphi(w)}\right\|_{\mathcal{Z}}=\lim _{|\varphi(w)| \rightarrow 1}\left\|D_{\varphi, u}^{n} h_{\varphi(w)}\right\|_{\mathcal{Z}}=0
$$


where

$$
\begin{aligned}
& f_{\varphi(w)}(z)=\frac{1-|\varphi(w)|^{2}}{1-\overline{\varphi(w)} z}, \quad g_{\varphi(w)}(z)=\frac{\left(1-|\varphi(w)|^{2}\right)^{2}}{(1-\overline{\varphi(w)} z)^{2}} \\
& h_{\varphi(w)}(z)=\frac{\left(1-|\varphi(w)|^{2}\right)^{3}}{(1-\overline{\varphi(w)} z)^{3}}, \quad z \in \mathbb{D} .
\end{aligned}
$$

(c)

$$
\begin{aligned}
\lim _{|\varphi(z)| \rightarrow 1} \frac{\left(1-|z|^{2}\right)\left|u^{\prime \prime}(z)\right|}{\left(1-|\varphi(z)|^{2}\right)^{n}} & =\lim _{|\varphi(z)| \rightarrow 1} \frac{\left(1-|z|^{2}\right)|u(z)|\left|\varphi^{\prime}(z)\right|^{2}}{\left(1-|\varphi(z)|^{2}\right)^{n+2}} \\
& =\lim _{|\varphi(z)| \rightarrow 1} \frac{\left(1-|z|^{2}\right)\left|2 u^{\prime}(z) \varphi^{\prime}(z)+u(z) \varphi^{\prime \prime}(z)\right|}{\left(1-|\varphi(z)|^{2}\right)^{1+n}}=0 .
\end{aligned}
$$

Motivated by these observations, the purpose of this paper is to give some estimates of the essential norm for the operator $D_{\varphi, u}^{n}: \mathcal{B} \rightarrow \mathcal{Z}$. Moreover, we give a new characterization for the boundedness, compactness, and essential norm of the operator $D_{\varphi, u}^{n}: \mathcal{B} \rightarrow \mathcal{Z}$.

Throughout this paper, we say that $P \lesssim Q$ if there exists a constant $C$ such that $P \leq C Q$. The symbol $P \approx Q$ means that $P \lesssim Q \lesssim P$.

\section{Essential norm of $D_{\varphi, u}^{n}: \mathcal{B} \rightarrow \mathcal{Z}$}

In this section, we give two estimates of the essential norm for the operator $D_{\varphi, u}^{n}: \mathcal{B} \rightarrow \mathcal{Z}$.

Theorem 2.1 Let $n$ be a positive integer, $u \in H(\mathbb{D})$, and $\varphi$ be an analytic self-map of $\mathbb{D}$ such that $D_{\varphi, u}^{n}: \mathcal{B} \rightarrow \mathcal{Z}$ is bounded. Then

$$
\left\|D_{\varphi, u}^{n}\right\|_{e, \mathcal{B} \rightarrow \mathcal{Z}} \approx \max \{A, B, C\} \approx \max \{E, F, G\}
$$

where

$$
\begin{aligned}
& A:=\underset{|a| \rightarrow 1}{\limsup }\left\|D_{\varphi, u}^{n}\left(\frac{1-|a|^{2}}{1-\bar{a} z}\right)\right\|_{\mathcal{Z}}, \quad B:=\limsup _{|a| \rightarrow 1}\left\|D_{\varphi, u}^{n}\left(\frac{\left(1-|a|^{2}\right)^{2}}{(1-\bar{a} z)^{2}}\right)\right\|_{\mathcal{Z}}, \\
& C:=\limsup _{|a| \rightarrow 1}\left\|D_{\varphi, u}^{n}\left(\frac{\left(1-|a|^{2}\right)^{3}}{(1-\bar{a} z)^{3}}\right)\right\|_{\mathcal{Z}}, \quad F:=\limsup _{|\varphi(z)| \rightarrow 1} \frac{\left(1-|z|^{2}\right)\left|u^{\prime \prime}(z)\right|}{\left(1-|\varphi(z)|^{2}\right)^{n}} \\
& E:=\limsup _{|\varphi(z)| \rightarrow 1} \frac{\left(1-|z|^{2}\right)\left|2 u^{\prime}(z) \varphi^{\prime}(z)+u(z) \varphi^{\prime \prime}(z)\right|}{\left(1-|\varphi(z)|^{2}\right)^{n+1}}
\end{aligned}
$$

and

$$
G:=\limsup _{|\varphi(z)| \rightarrow 1} \frac{\left(1-|z|^{2}\right)|u(z)|\left|\varphi^{\prime}(z)\right|^{2}}{\left(1-|\varphi(z)|^{2}\right)^{n+2}}
$$

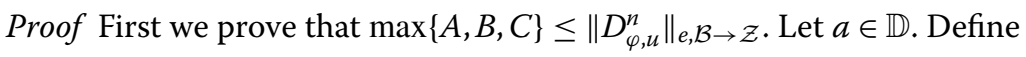

$$
f_{a}(z)=\frac{1-|a|^{2}}{(1-\bar{a} z)}, \quad g_{a}(z)=\frac{\left(1-|a|^{2}\right)^{2}}{(1-\bar{a} z)^{2}}, \quad h_{a}(z)=\frac{\left(1-|a|^{2}\right)^{3}}{(1-\bar{a} z)^{3}}, \quad z \in \mathbb{D} \text {. }
$$

It is easy to check that $f_{a}, g_{a}, h_{a} \in \mathcal{B}_{0}$ and $\left\|f_{a}\right\|_{\mathcal{B}} \lesssim 1,\left\|g_{a}\right\|_{\mathcal{B}} \lesssim 1,\left\|h_{a}\right\|_{\mathcal{B}} \lesssim 1$ for all $a \in \mathbb{D}$ and $f_{a}, g_{a}, h_{a}$ converge to 0 weakly in $\mathcal{B}$ as $|a| \rightarrow 1$. This follows since a bounded sequence 
contained in $\mathcal{B}_{0}$ which converges uniformly to 0 on compact subsets of $\mathbb{D}$ converges weakly to 0 in $\mathcal{B}$ (see [37, 42]). Thus, for any compact operator $K: \mathcal{B}_{0} \rightarrow \mathcal{Z}$, we have

$$
\lim _{|a| \rightarrow 1}\left\|K f_{a}\right\|_{\mathcal{Z}}=0, \quad \lim _{|a| \rightarrow 1}\left\|K g_{a}\right\|_{\mathcal{Z}}=0, \quad \lim _{|a| \rightarrow 1}\left\|K h_{a}\right\|_{\mathcal{Z}}=0 .
$$

Hence

$$
\begin{aligned}
\left\|D_{\varphi, u}^{n}-K\right\|_{\mathcal{B} \rightarrow \mathcal{Z}} & \gtrsim \limsup _{|a| \rightarrow 1}\left\|\left(D_{\varphi, u}^{n}-K\right) f_{a}\right\|_{\mathcal{Z}} \\
& \geq \limsup _{|a| \rightarrow 1}\left\|D_{\varphi, u}^{n} f_{a}\right\|_{\mathcal{Z}}-\limsup _{|a| \rightarrow 1}\left\|K f_{a}\right\|_{\mathcal{Z}}=A, \\
\left\|D_{\varphi, u}^{n}-K\right\|_{\mathcal{B} \rightarrow \mathcal{Z}} & \gtrsim \limsup _{|a| \rightarrow 1}\left\|\left(D_{\varphi, u}^{n}-K\right) g_{a}\right\|_{\mathcal{Z}} \\
& \geq \limsup _{|a| \rightarrow 1}\left\|D_{\varphi, u}^{n} g_{a}\right\|_{\mathcal{Z}}-\limsup _{|a| \rightarrow 1}\left\|K g_{a}\right\|_{\mathcal{Z}}=B,
\end{aligned}
$$

and

$$
\begin{aligned}
\left\|D_{\varphi, u}^{n}-K\right\|_{\mathcal{B} \rightarrow \mathcal{Z}} & \gtrsim \limsup _{|a| \rightarrow 1}\left\|\left(D_{\varphi, u}^{n}-K\right) h_{a}\right\|_{\mathcal{Z}} \\
& \geq \limsup _{|a| \rightarrow 1}\left\|D_{\varphi, u}^{n} h_{a}\right\|_{\mathcal{Z}}-\limsup _{|a| \rightarrow 1}\left\|K h_{a}\right\|_{\mathcal{Z}}=C .
\end{aligned}
$$

Therefore, from the definition of the essential norm, we obtain

$$
\left\|D_{\varphi, u}^{n}\right\|_{e, \mathcal{B} \rightarrow \mathcal{Z}}=\inf _{K}\left\|D_{\varphi, u}^{n}-K\right\|_{\mathcal{B} \rightarrow \mathcal{Z}} \gtrsim \max \{A, B, C\}
$$

Next, we prove that $\left\|D_{\varphi, u}^{n}\right\|_{e, \mathcal{B} \rightarrow \mathcal{Z}} \gtrsim \max \{E, F, G\}$. Let $\left\{z_{j}\right\}_{j \in \mathbb{N}}$ be a sequence in $\mathbb{D}$ such that $\left|\varphi\left(z_{j}\right)\right| \rightarrow 1$ as $j \rightarrow \infty$. Define

$$
\begin{aligned}
& k_{j}(z)=\frac{1-\left|\varphi\left(z_{j}\right)\right|^{2}}{1-\overline{\varphi\left(z_{j}\right)} z}-\frac{2 n+5}{(n+1)(n+3)} \frac{\left(1-\left|\varphi\left(z_{j}\right)\right|^{2}\right)^{2}}{\left(1-\overline{\varphi\left(z_{j}\right)} z\right)^{2}}+\frac{2}{(n+1)(n+3)} \frac{\left(1-\left|\varphi\left(z_{j}\right)\right|^{2}\right)^{3}}{\left(1-\overline{\varphi\left(z_{j}\right)} z\right)^{3}}, \\
& l_{j}(z)=\frac{1-\left|\varphi\left(z_{j}\right)\right|^{2}}{1-\overline{\varphi\left(z_{j}\right)} z}-\frac{2(n+3)}{2+(n+1)(n+4)} \frac{\left(1-\left|\varphi\left(z_{j}\right)\right|^{2}\right)^{2}}{\left(1-\overline{\varphi\left(z_{j}\right)} z\right)^{2}}+\frac{2}{2+(n+1)(n+4)} \frac{\left(1-\left|\varphi\left(z_{j}\right)\right|^{2}\right)^{3}}{\left(1-\overline{\varphi\left(z_{j}\right)} z\right)^{3}}
\end{aligned}
$$

and

$$
m_{j}(z)=\frac{1-\left|\varphi\left(z_{j}\right)\right|^{2}}{1-\overline{\varphi\left(z_{j}\right)} z}-\frac{2}{n+1} \frac{\left(1-\left|\varphi\left(z_{j}\right)\right|^{2}\right)^{2}}{\left(1-\overline{\varphi\left(z_{j}\right)} z\right)^{2}}+\frac{2}{(n+1)(n+2)} \frac{\left(1-\left|\varphi\left(z_{j}\right)\right|^{2}\right)^{3}}{\left(1-\overline{\varphi\left(z_{j}\right)} z\right)^{3}} .
$$

Similarly to the above we see that all $k_{j}, l_{j}$, and $m_{j}$ belong to $\mathcal{B}_{0}$ and converge to 0 weakly in $\mathcal{B}$. Moreover,

$$
\begin{aligned}
& k_{j}^{(n)}\left(\varphi\left(z_{j}\right)\right)=0, \quad k_{j}^{(n+2)}\left(\varphi\left(z_{j}\right)\right)=0, \quad\left|k_{j}^{(n+1)}\left(\varphi\left(z_{j}\right)\right)\right|=\frac{n !}{n+3} \frac{\left|\varphi\left(z_{j}\right)\right|^{n+1}}{\left(1-\left|\varphi\left(z_{j}\right)\right|^{2}\right)^{n+1}}, \\
& l_{j}^{(n+1)}\left(\varphi\left(z_{j}\right)\right)=0, \quad l_{j}^{(n+2)}\left(\varphi\left(z_{j}\right)\right)=0, \quad\left|l_{j}^{(n)}\left(\varphi\left(z_{j}\right)\right)\right|=\frac{2 n !}{2+(n+1)(n+4)} \frac{\left|\varphi\left(z_{j}\right)\right|^{n}}{\left(1-\left|\varphi\left(z_{j}\right)\right|^{2}\right)^{n}}, \\
& m_{j}^{(n)}\left(\varphi\left(z_{j}\right)\right)=0, \quad m_{j}^{(n+1)}\left(\varphi\left(z_{j}\right)\right)=0, \quad\left|m_{j}^{(n+2)}\left(\varphi\left(z_{j}\right)\right)\right|=2 n ! \frac{\left|\varphi\left(z_{j}\right)\right|^{n+2}}{\left(1-\left|\varphi\left(z_{j}\right)\right|^{2}\right)^{n+2}} .
\end{aligned}
$$


Then for any compact operator $K: \mathcal{B} \rightarrow \mathcal{Z}$, we obtain

$$
\begin{aligned}
\left\|D_{\varphi, u}^{n}-K\right\|_{\mathcal{B} \rightarrow \mathcal{Z}} & \gtrsim \limsup _{j \rightarrow \infty}\left\|D_{\varphi, u}^{n}\left(k_{j}\right)\right\|_{\mathcal{Z}}-\limsup _{j \rightarrow \infty}\left\|K\left(k_{j}\right)\right\|_{\mathcal{Z}} \\
& \gtrsim \limsup _{j \rightarrow \infty} \frac{\left(1-\left|z_{j}\right|^{2}\right)\left|2 u^{\prime}\left(z_{j}\right) \varphi^{\prime}\left(z_{j}\right)+u\left(z_{j}\right) \varphi^{\prime \prime}\left(z_{j}\right) \| \varphi\left(z_{j}\right)\right|^{n+1}}{\left(1-\left|\varphi\left(z_{j}\right)\right|^{2}\right)^{n+1}}, \\
\left\|D_{\varphi, u}^{n}-K\right\|_{\mathcal{B} \rightarrow \mathcal{Z}} & \gtrsim \limsup _{j \rightarrow \infty}\left\|D_{\varphi, u}^{n}\left(l_{j}\right)\right\|_{\mathcal{Z}}-\limsup _{j \rightarrow \infty}\left\|K\left(l_{j}\right)\right\|_{\mathcal{Z}} \\
& \gtrsim \limsup _{j \rightarrow \infty} \frac{\left(1-\left|z_{j}\right|^{2}\right)\left|u^{\prime \prime}\left(z_{j}\right) \| \varphi\left(z_{j}\right)\right|^{n}}{\left(1-\left|\varphi\left(z_{j}\right)\right|^{2}\right)^{n}}
\end{aligned}
$$

and

$$
\begin{aligned}
\left\|D_{\varphi, u}^{n}-K\right\|_{\mathcal{B} \rightarrow \mathcal{Z}} & \gtrsim \limsup _{j \rightarrow \infty}\left\|D_{\varphi, u}^{n}\left(m_{j}\right)\right\|_{\mathcal{Z}}-\limsup _{j \rightarrow \infty}\left\|K\left(m_{j}\right)\right\|_{\mathcal{Z}} \\
& \gtrsim \limsup _{j \rightarrow \infty} \frac{\left(1-\left|z_{j}\right|^{2}\right)\left|u\left(z_{j}\right) \| \varphi^{\prime}\left(z_{j}\right)\right|^{2}\left|\varphi\left(z_{j}\right)\right|^{n+2}}{\left(1-\left|\varphi\left(z_{j}\right)\right|^{2}\right)^{n+2}} .
\end{aligned}
$$

From the definition of the essential norm, we obtain

$$
\begin{aligned}
\left\|D_{\varphi, u}^{n}\right\|_{e, \mathcal{B} \rightarrow \mathcal{Z}} & =\inf _{K}\left\|D_{\varphi, u}^{n}-K\right\|_{\mathcal{B} \rightarrow \mathcal{Z}} \\
& \gtrsim \limsup _{j \rightarrow \infty} \frac{\left(1-\left|z_{j}\right|^{2}\right)\left|2 u^{\prime}\left(z_{j}\right) \varphi^{\prime}\left(z_{j}\right)+u\left(z_{j}\right) \varphi^{\prime \prime}\left(z_{j}\right)\right|\left|\varphi\left(z_{j}\right)\right|^{n+1}}{\left(1-\left|\varphi\left(z_{j}\right)\right|^{2}\right)^{n+1}} \\
& =\limsup _{|\varphi(z)| \rightarrow 1} \frac{\left(1-|z|^{2}\right)\left|2 u^{\prime}(z) \varphi^{\prime}(z)+u(z) \varphi^{\prime \prime}(z)\right|}{\left(1-|\varphi(z)|^{2}\right)^{n+1}}=E, \\
\left\|D_{\varphi, u}^{n}\right\|_{e, \mathcal{B} \rightarrow \mathcal{Z}} & =\inf _{K}\left\|D_{\varphi, u}^{n}-K\right\|_{\mathcal{B} \rightarrow \mathcal{Z}} \\
& \gtrsim \limsup _{j \rightarrow \infty} \frac{\left(1-\left|z_{j}\right|^{2}\right)\left|u^{\prime \prime}\left(z_{j}\right)\right|\left|\varphi\left(z_{j}\right)\right|^{n}}{\left(1-\left|\varphi\left(z_{j}\right)\right|^{2}\right)^{n}} \\
& =\limsup _{|\varphi(z)| \rightarrow 1} \frac{\left(1-|z|^{2}\right)\left|u^{\prime \prime}(z)\right|}{\left(1-|\varphi(z)|^{2}\right)^{n}}=F,
\end{aligned}
$$

and

$$
\begin{aligned}
\left\|D_{\varphi, u}^{n}\right\|_{e, \mathcal{B} \rightarrow \mathcal{Z}} & =\inf _{K}\left\|D_{\varphi, u}^{n}-K\right\|_{\mathcal{B} \rightarrow \mathcal{Z}} \\
& \gtrsim \limsup _{j \rightarrow \infty} \frac{\left(1-\left|z_{j}\right|^{2}\right)\left|u\left(z_{j}\right) \| \varphi^{\prime}\left(z_{j}\right)\right|^{2}\left|\varphi\left(z_{j}\right)\right|^{n+2}}{\left(1-\left|\varphi\left(z_{j}\right)\right|^{2}\right)^{n+2}} \\
& =\limsup _{|\varphi(z)| \rightarrow 1} \frac{\left(1-|z|^{2}\right)|u(z)|\left|\varphi^{\prime}(z)\right|^{2}}{\left(1-|\varphi(z)|^{2}\right)^{n+2}}=G .
\end{aligned}
$$

Hence

$$
\left\|D_{\varphi, u}^{n}\right\|_{e, \mathcal{B} \rightarrow \mathcal{Z}} \gtrsim \max \{E, F, G\} .
$$


Now, we prove that

$$
\left\|D_{\varphi, u}^{n}\right\|_{e, \mathcal{B} \rightarrow \mathcal{Z}} \lesssim \max \{A, B, C\} \quad \text { and } \quad\left\|D_{\varphi, u}^{n}\right\|_{e, \mathcal{B} \rightarrow \mathcal{Z}} \lesssim \max \{E, F, G\}
$$

For $r \in[0,1)$, set $K_{r}: H(\mathbb{D}) \rightarrow H(\mathbb{D})$ by $\left(K_{r} f\right)(z)=f_{r}(z)=f(r z), f \in H(\mathbb{D})$. It is obvious that $f_{r} \rightarrow f$ uniformly on compact subsets of $\mathbb{D}$ as $r \rightarrow 1$. Moreover, the operator $K_{r}$ is compact on $\mathcal{B}$ and $\left\|K_{r}\right\|_{\mathcal{B} \rightarrow \mathcal{B}} \leq 1$ (see [37]). Let $\left\{r_{j}\right\} \subset(0,1)$ be a sequence such that $r_{j} \rightarrow 1$ as $j \rightarrow \infty$. Then for all positive integer $j$, the operator $D_{\varphi, u}^{n} K_{r_{j}}: \mathcal{B} \rightarrow \mathcal{Z}$ is compact. By the definition of the essential norm, we get

$$
\left\|D_{\varphi, u}^{n}\right\|_{e, \mathcal{B} \rightarrow \mathcal{Z}} \leq \limsup _{j \rightarrow \infty}\left\|D_{\varphi, u}^{n}-D_{\varphi, u}^{n} K_{r_{j}}\right\|_{\mathcal{B} \rightarrow \mathcal{Z}}
$$

Therefore, we only need to prove that

$$
\limsup _{j \rightarrow \infty}\left\|D_{\varphi, u}^{n}-D_{\varphi, u}^{n} K_{r_{j}}\right\|_{\mathcal{B} \rightarrow \mathcal{Z}} \lesssim \max \{A, B, C\}
$$

and

$$
\limsup _{j \rightarrow \infty}\left\|D_{\varphi, u}^{n}-D_{\varphi, u}^{n} K_{r_{j}}\right\|_{\mathcal{B} \rightarrow \mathcal{Z}} \lesssim \max \{E, F, G\}
$$

For any $f \in \mathcal{B}$ such that $\|f\|_{\mathcal{B}} \leq 1$, we consider

$$
\begin{aligned}
&\left\|\left(D_{\varphi, u}^{n}-D_{\varphi, u}^{n} K_{r_{j}}\right) f\right\|_{\mathcal{Z}} \\
&=\mid u(0) f^{(n)}(\varphi(0))-r_{j}^{n} u(0) f^{(n)}\left(r_{j} \varphi(0)\right) \mid \\
& \quad+\left|u^{\prime}(0)\left(f-f_{r_{j}}\right)^{(n)}(\varphi(0))+u(0)\left(f-f_{r_{j}}\right)^{(n+1)}(\varphi(0)) \varphi^{\prime}(0)\right| \\
& \quad+\left\|u \cdot\left(f-f_{r_{j}}\right)^{(n)} \circ \varphi\right\|_{*^{\prime}}
\end{aligned}
$$

where $\|f\|_{*}=\sup _{z \in \mathbb{D}}\left(1-|z|^{2}\right)\left|f^{\prime \prime}(z)\right|$.

It is obvious that

$$
\lim _{j \rightarrow \infty}\left|u(0) f^{(n)}(\varphi(0))-r_{j}^{n} u(0) f^{(n)}\left(r_{j} \varphi(0)\right)\right|=0
$$

and

$$
\lim _{j \rightarrow \infty}\left|u^{\prime}(0)\left(f-f_{r_{j}}\right)^{(n)}(\varphi(0))+u(0)\left(f-f_{r_{j}}\right)^{(n+1)}(\varphi(0)) \varphi^{\prime}(0)\right|=0 .
$$

Now, we consider

$$
\begin{aligned}
& \limsup _{j \rightarrow \infty}\left\|u \cdot\left(f-f_{r_{j}}\right)^{(n)} \circ \varphi\right\|_{*} \\
& \leq \limsup _{j \rightarrow \infty} \sup _{|\varphi(z)| \leq r_{N}}\left(1-|z|^{2}\right)\left|\left(f-f_{r_{j}}\right)^{(n+1)}(\varphi(z))\right|\left|2 u^{\prime}(z) \varphi^{\prime}(z)+u(z) \varphi^{\prime \prime}(z)\right| \\
& \quad+\limsup _{j \rightarrow \infty} \sup _{|\varphi(z)|>r_{N}}\left(1-|z|^{2}\right)\left|\left(f-f_{r_{j}}\right)^{(n+1)}(\varphi(z))\right|\left|2 u^{\prime}(z) \varphi^{\prime}(z)+u(z) \varphi^{\prime \prime}(z)\right|
\end{aligned}
$$




$$
\begin{aligned}
& +\limsup _{j \rightarrow \infty} \sup _{|\varphi(z)| \leq r_{N}}\left(1-|z|^{2}\right)\left|\left(f-f_{r_{j}}\right)^{(n)}(\varphi(z))\right|\left|u^{\prime \prime}(z)\right| \\
& +\limsup _{j \rightarrow \infty} \sup _{|\varphi(z)|>r_{N}}\left(1-|z|^{2}\right)\left|\left(f-f_{r_{j}}\right)^{(n)}(\varphi(z))\right|\left|u^{\prime \prime}(z)\right| \\
& +\limsup _{j \rightarrow \infty} \sup _{|\varphi(z)| \leq r_{N}}\left(1-|z|^{2}\right)\left|\left(f-f_{r_{j}}\right)^{(n+2)}(\varphi(z))\right|\left|\varphi^{\prime}(z)\right|^{2}|u(z)| \\
& +\limsup _{j \rightarrow \infty} \sup _{|\varphi(z)|>r_{N}}\left(1-|z|^{2}\right)\left|\left(f-f_{r_{j}}\right)^{(n+2)}(\varphi(z))\right|\left|\varphi^{\prime}(z)\right|^{2}|u(z)| \\
& =Q_{1}+Q_{2}+Q_{3}+Q_{4}+Q_{5}+Q_{6},
\end{aligned}
$$

where $N \in \mathbb{N}$ is large enough such that $r_{j} \geq \frac{1}{2}$ for all $j \geq N$,

$$
\begin{aligned}
& Q_{1}:=\limsup _{j \rightarrow \infty} \sup _{|\varphi(z)| \leq r_{N}}\left(1-|z|^{2}\right)\left|\left(f-f_{r_{j}}\right)^{(n+1)}(\varphi(z))\right|\left|2 u^{\prime}(z) \varphi^{\prime}(z)+u(z) \varphi^{\prime \prime}(z)\right|, \\
& Q_{2}:=\limsup _{j \rightarrow \infty} \sup _{|\varphi(z)|>r_{N}}\left(1-|z|^{2}\right)\left|\left(f-f_{r_{j}}\right)^{(n+1)}(\varphi(z))\right|\left|2 u^{\prime}(z) \varphi^{\prime}(z)+u(z) \varphi^{\prime \prime}(z)\right|, \\
& Q_{3}:=\limsup _{j \rightarrow \infty} \sup _{|\varphi(z)| \leq r_{N}}\left(1-|z|^{2}\right)\left|\left(f-f_{r_{j}}\right)^{(n)}(\varphi(z))\right|\left|u^{\prime \prime}(z)\right|, \\
& Q_{4}:=\underset{j \rightarrow \infty}{\limsup \sup _{|\varphi(z)|>r_{N}}\left(1-|z|^{2}\right)\left|\left(f-f_{r_{j}}\right)^{(n)}(\varphi(z))\right|\left|u^{\prime \prime}(z)\right|,} \\
& Q_{5}:=\limsup _{j \rightarrow \infty} \sup _{|\varphi(z)| \leq r_{N}}\left(1-|z|^{2}\right)\left|\left(f-f_{r_{j}}\right)^{(n+2)}(\varphi(z))\right|\left|\varphi^{\prime}(z)\right|^{2}|u(z)|,
\end{aligned}
$$

and

$$
Q_{6}:=\limsup _{j \rightarrow \infty} \sup _{|\varphi(z)|>r_{N}}\left(1-|z|^{2}\right)\left|\left(f-f_{r_{j}}\right)^{(n+2)}(\varphi(z))\right|\left|\varphi^{\prime}(z)\right|^{2}|u(z)| .
$$

Since $D_{\varphi, u}^{n}: \mathcal{B} \rightarrow \mathcal{Z}$ is bounded, by Theorem 1 of [12], we see that $u \in \mathcal{Z}$,

$$
\widetilde{K}_{1}:=\sup _{z \in \mathbb{D}}\left(1-|z|^{2}\right)\left|2 u^{\prime}(z) \varphi^{\prime}(z)+u(z) \varphi^{\prime \prime}(z)\right|<\infty
$$

and

$$
\widetilde{K}_{2}:=\sup _{z \in \mathbb{D}}\left(1-|z|^{2}\right)\left|\varphi^{\prime}(z)\right|^{2}|u(z)|<\infty
$$

Since $r_{j}^{n+1} f_{r_{j}}^{(n+1)} \rightarrow f^{(n+1)}$, as well as $r_{j}^{n+2} f_{r_{j}}^{(n+2)} \rightarrow f^{(n+2)}$ uniformly on compact subsets of $\mathbb{D}$ as $j \rightarrow \infty$, we have

$$
Q_{1} \leq \widetilde{K}_{1} \limsup _{j \rightarrow \infty} \sup _{|w| \leq r_{N}}\left|f^{(n+1)}(w)-r_{j}^{n+1} f^{(n+1)}\left(r_{j} w\right)\right|=0
$$

and

$$
Q_{5} \leq \widetilde{K}_{2} \limsup _{j \rightarrow \infty} \sup _{|w| \leq r_{N}}\left|f^{(n+2)}(w)-r_{j}^{n+2} f^{(n+2)}\left(r_{j} w\right)\right|=0
$$


Similarly, from the fact that $u \in \mathcal{Z}$ we have

$$
Q_{3} \leq\|u\|_{\mathcal{Z}} \limsup _{j \rightarrow \infty} \sup _{|w| \leq r_{N}}\left|f^{(n)}(w)-r_{j}^{n} f^{(n)}\left(r_{j} w\right)\right|=0 .
$$

Next we consider $Q_{2}$. We have $Q_{2} \leq \lim \sup _{j \rightarrow \infty}\left(S_{1}+S_{2}\right)$, where

$$
S_{1}:=\sup _{|\varphi(z)|>r_{N}}\left(1-|z|^{2}\right)\left|f^{(n+1)}(\varphi(z))\right|\left|2 u^{\prime}(z) \varphi^{\prime}(z)+u(z) \varphi^{\prime \prime}(z)\right|
$$

and

$$
S_{2}:=\sup _{|\varphi(z)|>r_{N}}\left(1-|z|^{2}\right) r_{j}^{n+1}\left|f^{(n+1)}\left(r_{j} \varphi(z)\right)\right|\left|2 u^{\prime}(z) \varphi^{\prime}(z)+u(z) \varphi^{\prime \prime}(z)\right| .
$$

First we estimate $S_{1}$. Using the fact that $\|f\|_{\mathcal{B}} \leq 1$ and Theorem 5.4 in [31], we have

$$
\begin{aligned}
S_{1}= & \sup _{|\varphi(z)|>r_{N}}\left(1-|z|^{2}\right)\left|f^{(n+1)}(\varphi(z))\right|\left|2 u^{\prime}(z) \varphi^{\prime}(z)+u(z) \varphi^{\prime \prime}(z)\right| \\
& \times \frac{\left(1-|\varphi(z)|^{2}\right)^{n+1}(n+3)}{|\varphi(z)|^{n+1} n !} \frac{|\varphi(z)|^{n+1} n !}{(n+3)\left(1-|\varphi(z)|^{2}\right)^{n+1}} \\
\lesssim & \frac{(n+3)\|f\|_{\mathcal{B}}}{n ! r_{N}^{n+1}} \sup _{|\varphi(z)|>r_{N}}\left(1-|z|^{2}\right)\left|2 u^{\prime}(z) \varphi^{\prime}(z)+u(z) \varphi^{\prime \prime}(z)\right| \\
& \times \frac{n !|\varphi(z)|^{n+1}}{(n+3)\left(1-|\varphi(z)|^{2}\right)^{n+1}} \\
\lesssim & \sup _{|\varphi(z)|>r_{N}}\left(1-|z|^{2}\right)\left|2 u^{\prime}(z) \varphi^{\prime}(z)+u(z) \varphi^{\prime \prime}(z)\right| \frac{n !|\varphi(z)|^{n+1}}{(n+3)\left(1-|\varphi(z)|^{2}\right)^{n+1}} \\
\lesssim & \sup _{|a|>r_{N}}\left\|D_{\varphi, u}^{n}\left(f_{a}-\frac{(2 n+5) g_{a}}{(n+1)(n+3)}+\frac{2 h_{a}}{(n+1)(n+3)}\right)\right\|_{\mathcal{Z}} \\
\lesssim & \sup _{|a|>r_{N}}\left\|D_{\varphi, u}^{n}\left(f_{a}\right)\right\|_{\mathcal{Z}}+\frac{2 n+5}{(n+1)(n+3)} \sup _{|a|>r_{N}}\left\|D_{\varphi, u}^{n}\left(g_{a}\right)\right\|_{\mathcal{Z}} \\
& +\frac{2}{(n+1)(n+3)} \sup _{|a|>r_{N}}\left\|D_{\varphi, u}^{n}\left(h_{a}\right)\right\|_{\mathcal{Z}} .
\end{aligned}
$$

Taking the limit as $N \rightarrow \infty$ we obtain

$$
\begin{aligned}
\limsup _{j \rightarrow \infty} S_{1} \lesssim & \limsup _{|a| \rightarrow 1}\left\|D_{\varphi, u}^{n}\left(f_{a}\right)\right\|_{\mathcal{Z}}+\limsup _{|a| \rightarrow 1}\left\|D_{\varphi, u}^{n}\left(g_{a}\right)\right\|_{\mathcal{Z}} \\
& +\limsup _{|a| \rightarrow 1}\left\|D_{\varphi, u}^{n}\left(h_{a}\right)\right\|_{\mathcal{Z}} \\
= & A+B+C .
\end{aligned}
$$

Similarly, we have $\limsup _{j \rightarrow \infty} S_{2} \lesssim A+B+C$, i.e., we get

$$
Q_{2} \lesssim A+B+C \lesssim \max \{A, B, C\}
$$

From (2.9), we see that

$$
\limsup _{j \rightarrow \infty} S_{1} \lesssim \limsup _{|\varphi(z)| \rightarrow 1} \frac{\left(1-|z|^{2}\right)\left|2 u^{\prime}(z) \varphi^{\prime}(z)+u(z) \varphi^{\prime \prime}(z)\right|}{\left(1-|\varphi(z)|^{2}\right)^{n+1}}=E .
$$


Similarly we have $\lim \sup _{j \rightarrow \infty} S_{2} \lesssim E$. Therefore

$$
Q_{2} \lesssim E
$$

Next we consider $Q_{4}$. We have $Q_{4} \leq \lim \sup _{j \rightarrow \infty}\left(S_{3}+S_{4}\right)$, where

$$
S_{3}:=\sup _{|\varphi(z)|>r_{N}}\left(1-|z|^{2}\right)\left|f^{(n)}(\varphi(z))\right|\left|u^{\prime \prime}(z)\right|
$$

and

$$
S_{4}:=\sup _{|\varphi(z)|>r_{N}}\left(1-|z|^{2}\right) r_{j}^{n}\left|f^{(n)}\left(r_{j} \varphi(z)\right)\right|\left|u^{\prime \prime}(z)\right|
$$

After some calculation, we have

$$
\begin{aligned}
S_{3}= & \sup _{|\varphi(z)|>r_{N}}\left(1-|z|^{2}\right)\left|f^{(n)}(\varphi(z))\right|\left|u^{\prime \prime}(z)\right| \\
& \times \frac{\left(1-|\varphi(z)|^{2}\right)^{n}(2+(n+1)(n+4))}{2 n !|\varphi(z)|^{n}} \frac{2}{2+(n+1)(n+4)} \frac{n !|\varphi(z)|^{n}}{\left(1-|\varphi(z)|^{2}\right)^{n}} \\
\lesssim & \frac{2^{n}(2+(n+1)(n+4))}{2 n !}\|f\|_{\mathcal{B}} \sup _{|\varphi(z)|>r_{N}}\left(1-|z|^{2}\right)\left|u^{\prime \prime}(z)\right| \\
\lesssim & \sup _{|\varphi(z)|>r_{N}} \frac{2+(n+1)(n+4)}{2+(n+1)(n+4)} \frac{n !|\varphi(z)|^{n}}{\left(1-|\varphi(z)|^{2}\right)^{n}} \\
\lesssim & \sup _{|a|>r_{N}}\left\|D_{\varphi, u}^{n}\left(f_{a}\right)\right\|_{\mathcal{Z}}+\frac{2\left(1-|z|^{2}\right)\left|u^{\prime \prime}(z) \| \varphi(z)\right|^{n}}{2+(n+3)} \\
& +\frac{\left.\left.2(z)\right|^{2}\right)^{n}}{2+(n+1)(n+4)} \sup _{|a|>r_{N}}\left\|D_{\varphi, u}^{n}\left(h_{a}\right)\right\|_{\mathcal{Z}} \\
\lesssim & \sup _{|a|>r_{N}}\left\|D_{\varphi, u}^{n}\left(f_{a}\right)\right\|_{\mathcal{Z}}+\sup _{|a|>r_{N}}\left\|D_{\varphi, u}^{n}\left(g_{a}\right)\right\|_{\mathcal{Z}}+D_{\varphi, u}^{n}\left(g_{a}\right) \|_{\mathcal{Z}}
\end{aligned}
$$

Taking the limit as $N \rightarrow \infty$ we obtain

$$
\begin{aligned}
\limsup _{j \rightarrow \infty} S_{3} \lesssim & \limsup _{|a| \rightarrow 1}\left\|D_{\varphi, u}^{n}\left(f_{a}\right)\right\|_{\mathcal{Z}}+\limsup _{|a| \rightarrow 1}\left\|D_{\varphi, u}^{n}\left(g_{a}\right)\right\|_{\mathcal{Z}} \\
& +\underset{|a| \rightarrow 1}{\limsup }\left\|D_{\varphi, u}^{n}\left(h_{a}\right)\right\|_{\mathcal{Z}} \\
= & A+B+C .
\end{aligned}
$$

Similarly, we have $\limsup _{j \rightarrow \infty} S_{4} \lesssim A+B+C$, i.e., we get

$$
Q_{4} \lesssim A+B+C \lesssim \max \{A, B, C\}
$$

From (2.12), we see that

$$
\limsup _{j \rightarrow \infty} S_{3} \lesssim \limsup _{|\varphi(z)| \rightarrow 1} \frac{\left(1-|z|^{2}\right)^{\beta}\left|u^{\prime \prime}(z)\right|}{\left(1-|\varphi(z)|^{2}\right)^{n}}=F .
$$


Similarly we have $\lim \sup _{j \rightarrow \infty} S_{4} \lesssim F$. Therefore

$$
Q_{4} \lesssim F
$$

Finally we consider $Q_{6}$. We have $Q_{6} \leq \lim \sup _{j \rightarrow \infty}\left(S_{5}+S_{6}\right)$, where

$$
S_{5}:=\sup _{|\varphi(z)|>r_{N}}\left(1-|z|^{2}\right)\left|f^{(n+2)}(\varphi(z))\right|\left|\varphi^{\prime}(z)\right|^{2}|u(z)|
$$

and

$$
S_{6}:=\sup _{|\varphi(z)|>r_{N}}\left(1-|z|^{2}\right) r_{j}^{n+2}\left|f^{(n+2)}\left(r_{j} \varphi(z)\right)\right|\left|\varphi^{\prime}(z)\right|^{2}|u(z)| .
$$

After some calculation, we have

$$
\begin{aligned}
S_{5} & \lesssim \frac{2^{n+2}\|f\|_{\mathcal{B}}}{2 n !} \sup _{|\varphi(z)|>r_{N}}\left(1-|z|^{2}\right)\left|\varphi^{\prime}(z)\right|^{2}|u(z)| \frac{2 n !|\varphi(z)|^{n+2}}{\left(1-|\varphi(z)|^{2}\right)^{n+2}} \\
& \lesssim \frac{2^{n+2}}{2 n !} \sup _{|\varphi(z)|>r_{N}}\left(1-|z|^{2}\right)\left|\varphi^{\prime}(z)\right|^{2}|u(z)| \frac{2 n !|\varphi(z)|^{n+2}}{\left(1-|\varphi(z)|^{2}\right)^{n+2}} \\
& \lesssim \sup _{|a|>r_{N}}\left\|D_{\varphi, u}^{n}\left(f_{a}-\frac{2}{n+1} g_{a}+\frac{2}{(n+1)(n+2)} h_{a}\right)\right\|_{\mathcal{Z}} \\
& \lesssim \sup _{|a|>r_{N}}\left\|D_{\varphi, u}^{n}\left(f_{a}\right)\right\|_{\mathcal{Z}}+\frac{2}{n+1} \sup _{|a|>r_{N}}\left\|D_{\varphi, u}^{n}\left(g_{a}\right)\right\|_{\mathcal{Z}}+\frac{2}{(n+1)(n+2)} \sup _{|a|>r_{N}}\left\|D_{\varphi, u}^{n}\left(h_{a}\right)\right\|_{\mathcal{Z}} \\
& \leq \sup _{|a|>r_{N}}\left\|D_{\varphi, u}^{n}\left(f_{a}\right)\right\|_{\mathcal{Z}}+\sup _{|a|>r_{N}}\left\|D_{\varphi, u}^{n}\left(g_{a}\right)\right\|_{\mathcal{Z}}+\sup _{|a|>r_{N}}\left\|D_{\varphi, u}^{n}\left(h_{a}\right)\right\|_{\mathcal{Z}} \cdot
\end{aligned}
$$

Taking the limit as $N \rightarrow \infty$ we obtain

$$
\begin{aligned}
\limsup _{j \rightarrow \infty} S_{5} \lesssim & \limsup _{|a| \rightarrow 1}\left\|D_{\varphi, u}^{n}\left(f_{a}\right)\right\|_{\mathcal{Z}}+\limsup _{|a| \rightarrow 1}\left\|D_{\varphi, u}^{n}\left(g_{a}\right)\right\|_{\mathcal{Z}} \\
& +\underset{|a| \rightarrow 1}{\limsup \sup }\left\|D_{\varphi, u}^{n}\left(h_{a}\right)\right\|_{\mathcal{Z}} \\
= & A+B+C .
\end{aligned}
$$

Similarly, we have $\limsup _{j \rightarrow \infty} S_{6} \lesssim A+B+C$, i.e., we get

$$
Q_{6} \lesssim A+B+C \lesssim \max \{A, B, C\}
$$

From (2.15), we see that

$$
\limsup _{j \rightarrow \infty} S_{5} \lesssim \limsup _{|\varphi(z)| \rightarrow 1} \frac{\left(1-|z|^{2}\right)\left|\varphi^{\prime}(z)\right|^{2}|u(z)|}{\left(1-|\varphi(z)|^{2}\right)^{n+2}}=G .
$$

Similarly we have $\limsup _{j \rightarrow \infty} S_{6} \lesssim G$. Therefore

$$
Q_{6} \lesssim G
$$


Hence, by (2.2), (2.3), (2.4), (2.5), (2.6), (2.7), (2.8), (2.10), (2.13), and (2.16) we get

$$
\begin{aligned}
& \limsup _{j \rightarrow \infty}\left\|D_{\varphi, u}^{n}-D_{\varphi, u}^{n} K_{r_{j}}\right\|_{\mathcal{B} \rightarrow \mathcal{Z}} \\
& \quad=\limsup _{j \rightarrow \infty} \sup _{\|f\|_{\mathcal{B}} \leq 1}\left\|\left(D_{\varphi, u}^{n}-D_{\varphi, u}^{n} K_{r_{j}}\right) f\right\|_{\mathcal{Z}} \\
& \quad=\limsup _{j \rightarrow \infty} \sup _{\|f\|_{\mathcal{B}} \leq 1}\left\|u \cdot\left(f-f_{r_{j}}\right)^{(n)} \circ \varphi\right\|_{*} \lesssim \max \{A, B, C\} .
\end{aligned}
$$

Similarly, by (2.2), (2.3), (2.4), (2.5), (2.6), (2.7), (2.8), (2.11), (2.14), and (2.17) we get

$$
\limsup _{j \rightarrow \infty}\left\|D_{\varphi, u}^{n}-D_{\varphi, u}^{n} K_{r_{j}}\right\|_{\mathcal{B} \rightarrow \mathcal{Z}} \lesssim \max \{E, F, G\}
$$

Therefore, by (2.1), (2.18), and (2.19), we obtain

$$
\left\|D_{\varphi, u}^{n}\right\|_{e, \mathcal{B} \rightarrow \mathcal{Z}} \lesssim \max \{A, B, C\} \quad \text { and } \quad\left\|D_{\varphi, u}^{n}\right\|_{e, \mathcal{B} \rightarrow \mathcal{Z}} \lesssim \max \{E, F, G\}
$$

This completes the proof of Theorem 2.1.

\section{New characterization of $D_{\varphi, u}^{n}: \mathcal{B} \rightarrow \mathcal{Z}$}

In this section, we give a new characterization for the boundedness, compactness, and essential norm of the operator $D_{\varphi, u}^{n}: \mathcal{B} \rightarrow \mathcal{Z}$. For this purpose, we present some definitions and some lemmas which will be used later.

The weighted space, denoted by $H_{v}^{\infty}$, consists of all $f \in H(\mathbb{D})$ such that

$$
\|f\|_{\nu}=\sup _{z \in \mathbb{D}} v(z)|f(z)|<\infty
$$

where $v: \mathbb{D} \rightarrow R_{+}$is a continuous, strictly positive, and bounded function. $H_{v}^{\infty}$ is a Banach space under the norm $\|\cdot\|_{v}$. The weighted $v$ is called radial if $v(z)=v(|z|)$ for all $z \in \mathbb{D}$. The associated weight $\tilde{v}$ of $v$ is as follows:

$$
\tilde{v}=\left(\sup \left\{|f(z)|: f \in H_{v}^{\infty},\|f\|_{v} \leq 1\right\}\right)^{-1}, \quad z \in \mathbb{D} .
$$

When $v=v_{\alpha}(z)=\left(1-|z|^{2}\right)^{\alpha}(0<\alpha<\infty)$, it is well known that $\tilde{v}_{\alpha}(z)=v_{\alpha}(z)$. In this case, we denote $H_{v}^{\infty}$ by $H_{v_{\alpha}}^{\infty}$.

Lemma 3.1 [33] For $\alpha>0$, we have $\lim _{k \rightarrow \infty} k^{\alpha}\left\|z^{k-1}\right\|_{v_{\alpha}}=\left(\frac{2 \alpha}{e}\right)^{\alpha}$.

Lemma 3.2 [51] Let $v$ and w be radial, non-increasing weights tending to zero at the boundary of $\mathbb{D}$. Then the following statements hold.

(a) The weighted composition operator $u C_{\varphi}: H_{v}^{\infty} \rightarrow H_{w}^{\infty}$ is bounded if and only if $\sup _{z \in \mathbb{D}} \frac{w(z)}{\tilde{v}(\varphi(z))}|u(z)|<\infty$. Moreover, the following holds:

$$
\left\|u C_{\varphi}\right\|_{H_{v}^{\infty} \rightarrow H_{w}^{\infty}}=\sup _{z \in \mathbb{D}} \frac{w(z)}{\tilde{v}(\varphi(z))}|u(z)|
$$


(b) Suppose $u C_{\varphi}: H_{v}^{\infty} \rightarrow H_{w}^{\infty}$ is bounded. Then

$$
\left\|u C_{\varphi}\right\|_{e, H_{\nu}^{\infty} \rightarrow H_{W}^{\infty}}=\lim _{s \rightarrow 1^{-}} \sup _{|\varphi(z)|>s} \frac{w(z)}{\tilde{v}(\varphi(z))}|u(z)| .
$$

Lemma 3.3 [52] Let $v$ and $w$ be radial, non-increasing weights tending to zero at the boundary of $\mathbb{D}$. Then the following statements hold.

(a) $u C_{\varphi}: H_{v}^{\infty} \rightarrow H_{w}^{\infty}$ is bounded if and only if $\sup _{k \geq 0} \frac{\left\|u \varphi^{k}\right\|_{w}}{\left\|z^{k}\right\|_{v}}<\infty$, with the norm comparable to the above supremum.

(b) Suppose $u C_{\varphi}: H_{v}^{\infty} \rightarrow H_{w}^{\infty}$ is bounded. Then

$$
\left\|u C_{\varphi}\right\|_{e, H_{v}^{\infty} \rightarrow H_{w}^{\infty}}=\limsup _{k \rightarrow \infty} \frac{\left\|u \varphi^{k}\right\|_{w}}{\left\|z^{k}\right\|_{v}} .
$$

Theorem 3.1 Let $n$ be a positive integer, $u \in H(\mathbb{D})$, and $\varphi$ be an analytic self-map of $\mathbb{D}$. Then the operator $D_{\varphi, u}^{n}: \mathcal{B} \rightarrow \mathcal{Z}$ is bounded if and only if

$$
\left\{\begin{array}{l}
\sup _{j \geq 1} j^{n+1}\left\|\left(2 u^{\prime} \varphi^{\prime}+u \varphi^{\prime \prime}\right) \varphi^{j-1}\right\|_{v_{1}}<\infty \\
\sup _{j \geq 1} j^{n}\left\|u^{\prime \prime} \varphi^{j-1}\right\|_{v_{1}<\infty} \\
\sup _{j \geq 1} j^{n+2}\left\|u \varphi^{\prime 2} \varphi^{j-1}\right\|_{v_{1}}<\infty
\end{array}\right.
$$

Proof By [16], $D_{\varphi, u}^{n}: \mathcal{B} \rightarrow \mathcal{Z}$ is bounded if and only if

$$
\left\{\begin{array}{l}
\sup _{z \in \mathbb{D}} \frac{\left(1-|z|^{2}\right)\left|2 u^{\prime}(z) \varphi^{\prime}(z)+u(z) \varphi^{\prime \prime}(z)\right|}{\left(1-|\varphi(z)|^{2}\right)^{n+1}}<\infty \\
\sup _{z \in \mathbb{D}} \frac{\left.(1-|z|)^{2}||\right|^{\prime \prime}(z) \mid}{\left(1-|(z)|^{2}\right)^{n}}<\infty \\
\sup _{z \in \mathbb{D}} \frac{\left(1-|z|^{2}\right)|u(z)|\left|\varphi^{\prime}(z)\right|^{2}}{\left(1-|\varphi(z)|^{2}\right)^{2+2}}<\infty
\end{array}\right.
$$

By Lemma 3.2, the first inequality in (3.2) is equivalent to the weighted composition operator $\left(2 u^{\prime} \varphi^{\prime}+u \varphi^{\prime \prime}\right) C_{\varphi}: H_{v_{n+1}}^{\infty} \rightarrow H_{v_{1}}^{\infty}$ is bounded. By Lemma 3.3, this is equivalent to

$$
\sup _{j \geq 1} \frac{\left\|\left(2 u^{\prime} \varphi^{\prime}+u \varphi^{\prime \prime}\right) \varphi^{j-1}\right\|_{\nu_{1}}}{\left\|z^{j-1}\right\|_{v_{1+n}}}<\infty
$$

The second inequality in (3.2) is equivalent to the operator $u^{\prime \prime} C_{\varphi}: H_{v_{n}}^{\infty} \rightarrow H_{v_{1}}^{\infty}$ is bounded. By Lemma 3.3, this is equivalent to

$$
\sup _{j \geq 1} \frac{\left\|u^{\prime \prime} \varphi^{j-1}\right\|_{\nu_{1}}}{\left\|z^{j-1}\right\|_{v_{n}}}<\infty .
$$

The third inequality in (3.2) is equivalent to the operator $u \varphi^{\prime 2} C_{\varphi}: H_{v_{n+2}}^{\infty} \rightarrow H_{v_{1}}^{\infty}$ is bounded. By Lemma 3.3, this is equivalent to

$$
\sup _{j \geq 1} \frac{\left\|u \varphi^{\prime 2} \varphi^{j-1}\right\|_{v_{1}}}{\left\|z^{j-1}\right\|_{v_{n+2}}}<\infty .
$$

By Lemma 3.1, we see that $D_{\varphi, u}^{n}: \mathcal{B} \rightarrow \mathcal{Z}$ is bounded if and only if

$$
\sup _{j \geq 1} j^{n+1}\left\|\left(2 u^{\prime} \varphi^{\prime}+u \varphi^{\prime \prime}\right) \varphi^{j-1}\right\|_{v_{1}} \approx \sup _{j \geq 1} \frac{j^{n+1}\left\|\left(2 u^{\prime} \varphi^{\prime}+u \varphi^{\prime \prime}\right) \varphi^{j-1}\right\|_{\nu_{1}}}{j^{n+1}\left\|z^{j-1}\right\|_{v_{1+n}}}<\infty,
$$




$$
\sup _{j \geq 1}^{n}\left\|u^{\prime \prime} \varphi^{j-1}\right\|_{v_{1}} \approx \sup _{j \geq 1} \frac{j^{n}\left\|u^{\prime \prime} \varphi^{j-1}\right\|_{\nu_{1}}}{j^{n}\left\|z^{j-1}\right\|_{v_{n}}}<\infty
$$

and

$$
\sup _{j \geq 1}^{n+2}\left\|u \varphi^{\prime 2} \varphi^{j-1}\right\|_{v_{1}} \approx \sup _{j \geq 1} \frac{j^{n+2}\left\|u \varphi^{\prime 2} \varphi^{j-1}\right\|_{v_{1}}}{j^{n+2}\left\|z^{j-1}\right\|_{v_{n+2}}}<\infty .
$$

The proof is completed.

Theorem 3.2 Let $n$ be a positive integer, $u \in H(\mathbb{D})$, and $\varphi$ be an analytic self-map of $\mathbb{D}$ such that $D_{\varphi, u}^{n}: \mathcal{B} \rightarrow \mathcal{Z}$ is bounded. Then

$$
\left\|D_{\varphi, u}^{n}\right\|_{e, \mathcal{B} \rightarrow \mathcal{Z}} \approx \max \left\{M_{1}, M_{2}, M_{3}\right\}
$$

where

$$
\begin{aligned}
& M_{1}:=\limsup _{j \rightarrow \infty} j^{1+n}\left\|\left(2 u^{\prime} \varphi^{\prime}+u \varphi^{\prime \prime}\right) \varphi^{j-1}\right\|_{\nu_{1}}, \\
& M_{2}:=\limsup _{j \rightarrow \infty} j^{n}\left\|u^{\prime \prime} \varphi^{j-1}\right\|_{\nu_{1}}, \quad M_{3}:=\limsup _{j \rightarrow \infty} j^{n+2}\left\|u\left(\varphi^{\prime}\right)^{2} \varphi^{j-1}\right\|_{\nu_{1}} .
\end{aligned}
$$

Proof From the proof of Theorem 3.1 we know that the boundedness of $D_{\varphi, u}^{n}: \mathcal{B} \rightarrow \mathcal{Z}$ is equivalent to the boundedness of the operators $\left(2 u^{\prime} \varphi^{\prime}+u \varphi^{\prime \prime}\right) C_{\varphi}: H_{v_{1+n}}^{\infty} \rightarrow H_{v_{1}}^{\infty}, u^{\prime \prime} C_{\varphi}$ : $H_{v_{n}}^{\infty} \rightarrow H_{v_{1}}^{\infty}$, and $u \varphi^{\prime 2} C_{\varphi}: H_{v_{n+2}}^{\infty} \rightarrow H_{v_{1}}^{\infty}$.

The upper estimate. By Lemmas 3.1 and 3.3, we get

$$
\begin{aligned}
& \left\|\left(2 u^{\prime} \varphi^{\prime}+u \varphi^{\prime \prime}\right) C_{\varphi}\right\|_{e, H_{v_{1+n}}^{\infty} \rightarrow H_{v_{1}}^{\infty}}=\limsup _{j \rightarrow \infty} \frac{\left\|\left(2 u^{\prime} \varphi^{\prime}+u \varphi^{\prime \prime}\right) \varphi^{j-1}\right\|_{\nu_{1}}}{\left\|z^{j-1}\right\|_{v_{1+n}}} \\
& =\limsup _{j \rightarrow \infty} \frac{j^{1+n}\left\|\left(2 u^{\prime} \varphi^{\prime}+u \varphi^{\prime \prime}\right) \varphi^{j-1}\right\|_{\nu_{1}}}{j^{1+n}\left\|z^{j-1}\right\|_{v_{1+n}}} \\
& \approx \limsup _{j \rightarrow \infty} j^{1+n}\left\|\left(2 u^{\prime} \varphi^{\prime}+u \varphi^{\prime \prime}\right) \varphi^{j-1}\right\|_{\nu_{1}} \\
& \left\|u^{\prime \prime} C_{\varphi}\right\|_{e, H_{v_{n}}^{\infty} \rightarrow H_{v_{1}}^{\infty}}=\limsup _{j \rightarrow \infty} \frac{\left\|u^{\prime \prime} \varphi^{j-1}\right\|_{\nu_{1}}}{\left\|z^{j-1}\right\|_{v_{n}}}=\limsup _{j \rightarrow \infty} \frac{j^{\alpha+n-1}\left\|u^{\prime \prime} \varphi^{j-1}\right\|_{\nu_{1}}}{j^{\alpha+n-1}\left\|z^{j-1}\right\|_{v_{n}}} \\
& \approx \limsup _{j \rightarrow \infty} j^{n}\left\|u^{\prime \prime} \varphi^{j-1}\right\|_{v_{1}},
\end{aligned}
$$

and

$$
\begin{aligned}
\left\|u \varphi^{\prime 2} C_{\varphi}\right\|_{e, H_{v_{n+2}}^{\infty} \rightarrow H_{v_{1}}^{\infty}} & =\limsup _{j \rightarrow \infty} \frac{\left\|u \varphi^{\prime 2} \varphi^{j-1}\right\|_{v_{1}}}{\left\|z^{j-1}\right\|_{v_{n+2}}}=\limsup _{j \rightarrow \infty} \frac{j^{n+2}\left\|u \varphi^{\prime 2} \varphi^{j-1}\right\|_{v_{1}}}{j^{n+2}\left\|z^{j-1}\right\|_{v_{n+2}}} \\
& \approx \limsup _{j \rightarrow \infty} j^{n+2}\left\|u \varphi^{\prime 2} \varphi^{j-1}\right\|_{v_{1}} .
\end{aligned}
$$

It follows that

$$
\begin{aligned}
\left\|D_{\varphi, u}^{n}\right\|_{e, \mathcal{B} \rightarrow \mathcal{Z}} \lesssim & \left\|\left(2 u^{\prime} \varphi^{\prime}+u \varphi^{\prime \prime}\right) C_{\varphi}\right\|_{e, H_{v_{1+n}}^{\infty} \rightarrow H_{v_{1}}^{\infty}}+\left\|u^{\prime \prime} C_{\varphi}\right\|_{e, H_{v_{n}}^{\infty} \rightarrow H_{v_{1}}^{\infty}} \\
& +\left\|u \varphi^{\prime 2} C_{\varphi}\right\|_{e, H_{v_{n+2}}^{\infty} \rightarrow H_{v_{1}}^{\infty}} \\
\lesssim & \max \left\{M_{1}, M_{2}, M_{3}\right\} .
\end{aligned}
$$


The lower estimate. From Theorem 2.1, and Lemmas 3.1 and 3.2, we have

$$
\begin{aligned}
\left\|D_{\varphi, u}^{n}\right\|_{e, \mathcal{B} \rightarrow \mathcal{Z}} & \gtrsim E=\left\|\left(2 u^{\prime} \varphi^{\prime}+u \varphi^{\prime \prime}\right) C_{\varphi}\right\|_{e, H_{v_{1+n}}^{\infty} \rightarrow H_{v_{1}}^{\infty}} \\
& =\limsup _{j \rightarrow \infty} \frac{\left\|\left(2 u^{\prime} \varphi^{\prime}+u \varphi^{\prime \prime}\right) \varphi^{j-1}\right\|_{v_{1}}}{\left\|z^{j-1}\right\|_{v_{1+n}}} \\
& \approx \limsup _{j \rightarrow \infty} j^{\alpha+n}\left\|\left(2 u^{\prime} \varphi^{\prime}+u \varphi^{\prime \prime}\right) \varphi^{j-1}\right\|_{v_{1}}, \\
\left\|D_{\varphi, u}^{n}\right\|_{e, \mathcal{B} \rightarrow \mathcal{Z}} & \gtrsim F=\left\|u^{\prime \prime} C_{\varphi}\right\|_{e, H_{v_{n}}^{\infty} \rightarrow H_{v_{1}}^{\infty}}=\limsup _{j \rightarrow \infty} \frac{\left\|u^{\prime \prime} \varphi^{j-1}\right\|_{v_{1}}}{\left\|z^{j-1}\right\|_{v_{n}}} \\
& \approx \limsup _{j \rightarrow \infty} j^{\alpha+n-1}\left\|u^{\prime \prime} \varphi^{j-1}\right\|_{v_{1}},
\end{aligned}
$$

and

$$
\begin{aligned}
\left\|D_{\varphi, u}^{n}\right\|_{e, \mathcal{B} \rightarrow \mathcal{Z}} & \gtrsim G=\left\|u \varphi^{\prime 2} C_{\varphi}\right\|_{e, H_{v_{n+2}}^{\infty} \rightarrow H_{\nu_{1}}^{\infty}}=\limsup _{j \rightarrow \infty} \frac{\left\|u \varphi^{\prime 2} \varphi^{j-1}\right\|_{\nu_{1}}}{\left\|z^{j-1}\right\|_{v_{n+2}}} \\
& \approx \limsup _{j \rightarrow \infty} j^{n+2}\left\|u \varphi^{\prime 2} \varphi^{j-1}\right\|_{v_{1}} .
\end{aligned}
$$

Therefore $\left\|D_{\varphi, u}^{n}\right\|_{e, \mathcal{B} \rightarrow \mathcal{Z}} \gtrsim \max \left\{M_{1}, M_{2}, M_{3}\right\}$. This completes the proof.

From Theorem 3.2, we immediately get the following result.

Theorem 3.3 Let $n$ be a positive integer, $u \in H(\mathbb{D})$, and $\varphi$ be an analytic self-map of $\mathbb{D}$ such that $D_{\varphi, u}^{n}: \mathcal{B} \rightarrow \mathcal{Z}$ is bounded. Then $D_{\varphi, u}^{n}: \mathcal{B} \rightarrow \mathcal{Z}$ is compact if and only if

$$
\limsup _{j \rightarrow \infty} j^{1+n}\left\|\left(2 u^{\prime} \varphi^{\prime}+u \varphi^{\prime \prime}\right) \varphi^{j-1}\right\|_{\nu_{1}}=0, \quad \limsup _{j \rightarrow \infty} j^{n}\left\|u^{\prime \prime} \varphi^{j-1}\right\|_{\nu_{1}}=0
$$

and

$$
\limsup _{j \rightarrow \infty} j^{n+2}\left\|u\left(\varphi^{\prime}\right)^{2} \varphi^{j-1}\right\|_{v_{1}}=0
$$

\section{Conclusion}

The boundedness and compactness of $D_{\varphi, u}^{n}: \mathcal{B} \rightarrow \mathcal{Z}$ were characterized in [12] and [16]. In this paper, we give a new characterization for the boundedness and compactness of $D_{\varphi, u}^{n}: \mathcal{B} \rightarrow \mathcal{Z}$. Moreover, using the method in [21], we completely characterize the essential norm of $D_{\varphi, u}^{n}: \mathcal{B} \rightarrow \mathcal{Z}$.

\section{Competing interests}

The authors declare that they have no competing interests.

\section{Authors' contributions}

The four authors contributed equally to the writing of this paper. They read and approved the final version of the manuscript.

\section{Author details}

'Department of Mathematics, Shantou University, Shantou, Guangdong 515063, China. ${ }^{2}$ School of Statistics and Management, Shanghai University of Finance and Economics, Shanghai, 200433, China. ${ }^{3}$ Faculty of Information Technology, Macau University of Science and Technology, Avenida Wai Long, Taipa, Macau. ${ }^{4}$ Department of Mathematics, Jiaying University, Meizhou, Guangdong 515063, China. 


\section{Acknowledgements}

This project is partially supported by the Macao Science and Technology Development Fund (No. 083/2014/A2).

\section{Received: 19 January 2016 Accepted: 1 April 2016 Published online: 21 April 2016}

\section{References}

1. Hibschweiler, R, Portnoy, N: Composition followed by differentiation between Bergman and Hardy spaces. Rocky Mt. J. Math. 35, 843-855 (2005)

2. Li, S, Stević, S: Composition followed by differentiation between Bloch type spaces. J. Comput. Anal. Appl. 9, 195-205 (2007)

3. Li, S, Stević, S: Composition followed by differentiation between $H^{\infty}$ and $\alpha$-Bloch spaces. Houst. J. Math. 35, 327-340 (2009)

4. Li, S, Stević, S: Products of composition and differentiation operators from Zygmund spaces to Bloch spaces and Bers spaces. Appl. Math. Comput. 217, 3144-3154 (2010)

5. Stević, S: Norm and essential norm of composition followed by differentiation from $\alpha$-Bloch spaces to $H_{\mu}^{\infty}$. Appl. Math. Comput. 207, 225-229 (2009)

6. Stević, S: Products of composition and differentiation operators on the weighted Bergman space. Bull. Belg. Math. Soc. Simon Stevin 16, 623-635 (2009)

7. Stević, S: Composition followed by differentiation from $H^{\infty}$ and the Bloch space to $n$th weighted-type spaces on the unit disk. Appl. Math. Comput. 216, 3450-3458 (2010)

8. Stević, S: Characterizations of composition followed by differentiation between Bloch-type spaces. Appl. Math. Comput. 218, 4312-4316 (2011)

9. Yang, W: Products of composition differentiation operators from $\mathcal{Q}_{k}(p, q)$ spaces to Bloch-type spaces. Abstr. Appl. Anal. 2009, Article ID 741920 (2009)

10. Stević, S, Sharma, A: Iterated differentiation followed by composition from Bloch-type spaces to weighted BMOA spaces. Appl. Math. Comput. 218, 3574-3580 (2011)

11. Wu, Y, Wulan, H: Products of differentiation and composition operators on the Bloch space. Collect. Math. 63, 93-107 (2012)

12. $\mathrm{Li}, \mathrm{H}, \mathrm{Fu}, \mathrm{X}$ : A new characterization of generalized weighted composition operators from the Bloch space into the Zygmund space. J. Funct. Spaces Appl. 2013, Article ID 925901 (2013)

13. Li, S, Stević, S: Generalized weighted composition operators from $\alpha$-Bloch spaces into weighted-type spaces. J. Inequal. Appl. 2015, Article ID 265 (2015)

14. Stević, S: Weighted differentiation composition operators from mixed-norm spaces to weighted-type spaces. Appl. Math. Comput. 211, 222-233 (2009)

15. Stević, S: Weighted differentiation composition operators from mixed-norm spaces to the $n$th weighted-type space on the unit disk. Abstr. Appl. Anal. 2010, Article ID 246287 (2010)

16. Stević, S: Weighted differentiation composition operators from $H^{\infty}$ and Bloch spaces to $n$th weighted-type spaces on the unit disk. Appl. Math. Comput. 216, 3634-3641 (2010)

17. Yang, W, Zhu, X: Generalized weighted composition operators from area Nevanlinna spaces to Bloch-type spaces. Taiwan. J. Math. 16, 869-883 (2012)

18. Zhu, X: Products of differentiation, composition and multiplication from Bergman type spaces to Bers type space. Integral Transforms Spec. Funct. 18, 223-231 (2007)

19. Zhu, X: Generalized weighted composition operators on weighted Bergman spaces. Numer. Funct. Anal. Optim. 30, 881-893 (2009)

20. Zhu, X: Generalized weighted composition operators on Bloch-type spaces. J. Inequal. Appl. 2015, Article ID 59 (2015)

21. Zhu, X: Essential norm of generalized weighted composition operators on Bloch-type spaces. Appl. Math. Comput. $274,133-142(2016)$

22. Li, S, Stević, S: Generalized composition operators on Zygmund spaces and Bloch type spaces. J. Math. Anal. Appl. $338,1282-1295(2008)$

23. Li, S, Stević, S: Products of composition and integral type operators from $H^{\infty}$ to the Bloch space. Complex Var. Elliptic Equ. 53(5), 463-474 (2008)

24. Li, S, Stević, S: Composition followed by differentiation from mixed-norm spaces to $\alpha$-Bloch spaces. Sb. Math 199(12), 1847-1857 (2008)

25. Li, S, Stević, S: Products of integral-type operators and composition operators between Bloch-type spaces. J. Math. Anal. Appl. 349, 596-610 (2009)

26. Stević, S: On an integral-type operator from logarithmic Bloch-type and mixed-norm spaces to Bloch-type spaces. Nonlinear Anal. TMA 71, 6323-6342 (2009)

27. Stević, S: Products of integral-type operators and composition operators from the mixed norm space to Bloch-type spaces. Sib. Math. J. 50, 726-736 (2009)

28. Stević, S: On some integral-type operators between a general space and Bloch-type spaces. Appl. Math. Comput. $218,2600-2618(2011)$

29. Stević, S, Sharma, A, Bhat, A: Products of multiplication composition and differentiation operators on weighted Bergman spaces. Appl. Math. Comput. 217, 8115-8125 (2011)

30. Stević, S, Sharma, A, Bhat, A: Essential norm of products of multiplication composition and differentiation operators on weighted Bergman spaces. Appl. Math. Comput. 218, 2386-2397 (2011)

31. Zhu, K: Operator Theory in Function Spaces, 2nd edn. Am. Math. Soc., Providence (2007)

32. Cowen, C, Maccluer, B: Composition Operators on Spaces of Analytic Functions. CRC Press, Boca Raton (1995)

33. Hyvärinen, $\mathrm{O}$, Lindström, M: Estimates of essential norm of weighted composition operators between Bloch-type spaces. J. Math. Anal. Appl. 393, 38-44 (2012)

34. Li, S, Stević, S: Weighted composition operators from Bergman-type spaces into Bloch spaces. Proc. Indian Acad. Sci. Math. Sci. 117(3), 371-385 (2007) 
35. Li, S, Stević, S: Weighted composition operators between $H^{\infty}$ and $\alpha$-Bloch spaces in the unit ball. Taiwan. J. Math. 12, 1625-1639 (2008)

36. Li, S, Stević, S: Weighted composition operators from Zygmund spaces into Bloch spaces. Appl. Math. Comput. 206, 825-831 (2008)

37. MacCluer, B, Zhao, R: Essential norm of weighted composition operators between Bloch-type spaces. Rocky Mt. J. Math. 33, 1437-1458 (2003)

38. Madigan, K, Matheson, A: Compact composition operators on the Bloch space. Trans. Am. Math. Soc. 347, 2679-2687 (1995)

39. Manhas, J, Zhao, R: New estimates of essential norms of weighted composition operators between Bloch type spaces. J. Math. Anal. Appl. 389, 32-47 (2012)

40. Montes-Rodriguez, A: The essential norm of composition operators on the Bloch space. Pac. J. Math. 188, 339-351 (1999)

41. Ohno, S, Stroethoff, K, Zhao, R: Weighted composition operators between Bloch-type spaces. Rocky Mt. J. Math. 33, 191-215 (2003)

42. Stević, S: Essential norms of weighted composition operators from the $\alpha$-Bloch space to a weighted-type space on the unit ball. Abstr. Appl. Anal. 2008, Article ID 279691 (2008)

43. Tjani, M: Compact composition operators on some Möbius invariant Banach space. PhD dissertation, Michigan State University (1996)

44. Wulan, H, Zheng, D, Zhu, K: Compact composition operators on BMOA and the Bloch space. Proc. Am. Math. Soc 137, 3861-3868 (2009)

45. Zhao, R: Essential norms of composition operators between Bloch type spaces. Proc. Am. Math. Soc. 138, 2537-2546 (2010)

46. Choe, B, Koo, H, Smith, W: Composition operators on small spaces. Integral Equ. Oper. Theory 56, 357-380 (2006)

47. Duren, $P$ : Theory of $H^{P}$ Spaces. Academic Press, New York (1970)

48. Esmaeili, K, Lindström, M: Weighted composition operators between Zygmund type spaces and their essential norms. Integral Equ. Oper. Theory 75, 473-490 (2013)

49. Li, S, Stević, S: Volterra type operators on Zygmund spaces. J. Inequal. Appl. 2007, Article ID 32124 (2007)

50. Yu, Y, Liu, Y: Weighted differentiation composition operators from $H^{\infty}$ to Zygmund spaces. Integral Transforms Spec. Funct. 22, 507-520 (2011)

51. Montes-Rodriguez, A: Weighed composition operators on weighted Banach spaces of analytic functions. J. Lond Math. Soc. 61, 872-884 (2000)

52. Hyvärinen, O, Kemppainen, M, Lindström, M, Rautio, A, Saukko, E: The essential norm of weighted composition operators on weighted Banach spaces of analytic functions. Integral Equ. Oper. Theory 72, 151-157 (2012)

\section{Submit your manuscript to a SpringerOpen ${ }^{\ominus}$ journal and benefit from:}

- Convenient online submission

- Rigorous peer review

- Immediate publication on acceptance

- Open access: articles freely available online

- High visibility within the field

- Retaining the copyright to your article 